\title{
APPLICATIONS OF UNIFORM CONVEXITY OF NONCOMMUTATIVE $L^{p}$-SPACES
}

\author{
BY
}

HIDEKI KOSAKI ${ }^{1}$

\begin{abstract}
We consider noncommutative $L^{p}$-spaces, $1<p<\infty$, associated with a von Neumann algebra, which is not necessarily semifinite, and obtain some consequences of their uniform convexity. Among other results, we obtain (i) the norm continuity of the "absolute value part" map from each $L^{p}$-space onto its positive part; (ii) a certain continuity result on Radon-Nikodym derivatives in the context of positive cones introduced by $\mathrm{H}$. Araki; and (iii) the necessary and sufficient condition for certain $L^{p}$-norm inequalities to become equalities. Some dominated convergence theorems for a probability gage are also considered.
\end{abstract}

1. Introduction. After the development of the modular theory [27] one can construct noncommutative $L^{p}$-spaces associated with a von Neumann algebra without a trace $[3,8,10,12,14,15]$. (Full details of $[\mathbf{1 0}]$ are now found in [30].) Also it is now known [15] that these $L^{p}$-spaces are uniformly convex Banach spaces. The purpose of the paper is to obtain certain consequences of this uniform convexity, which will be explained below.

After some preliminaries and technical lemmas in $\S \S 2$ and 3, respectively, in $\S 4$ we

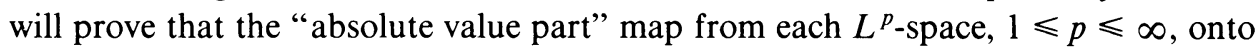
its positive part is norm-continuous. This continuity is known for $C_{p}$-ideals $[19,24]$, that is, the noncommutative $L^{p}$-spaces constructed from a factor of type $\mathbf{I}_{\infty}$. However, our proof is more technical because we have to deal with certain unbounded operators (see $\$ 2$ for details) instead of compact operators in $C_{p}$-ideals.

In $\S 5$ we will consider a one-parameter family $\left\{P^{\alpha}\right\}_{0 \leqslant \alpha \leqslant 1 / 2}$ of positive cones associated with a von Neumann algebra $\Re$ admitting a cyclic and separating vector $[1,3,13,14,16,25,27]$. We will prove that, for each $0 \leqslant \alpha \leqslant 1 / 4$, the map $\xi \in P^{\alpha} \rightarrow \omega_{\xi} \in \pi_{*}^{+}$is a homeomorphism with respect to the norm topologies, and that $0 \leqslant \alpha \leqslant 1 / 4$ can be replaced by $0 \leqslant \alpha \leqslant 1 / 2$ if $\Re$ is finite. The former is a consequence of the continuity result in $\S 4$, while the latter is a consequence of a certain dominated convergence theorem for a probability gage $[18,20,21,23,26,31]$ proved in $\$ 3$.

In $\S 6$, among other results, we will prove

$$
2^{1-p}\|a+b\|_{p}^{p} \leqslant\|a\|_{p}^{p}+\|b\|_{p}^{p} \leqslant\|a+b\|_{p}^{p}
$$

Received by the editors September 28, 1982 and, in revised form, May 16, 1983.

1980 Mathematics Subject Classification. Primary 46L10, 46L50; Secondary 47D25.

Key words and phrases. Noncommutative $L^{p}$-spaces associated with a von Neumann algebra, oneparameter family of positive cones, theory of gages, norm inequalities.

'Supported in part by the National Science Foundation (MCS-812158). 
for any $a, b$ in the positive part of each $L^{p}$-space, $1<p<\infty$. More importantly, we will also find when these inequalities and Clarkson's inequality become equalities.

The author wishes to thank the referee for useful comments. Indeed, the proof of Lemma 2.3 is due to the referee, and this greatly simplified lengthy arguments in the original version.

2. Noncommutative $L^{p}$-spaces. We collect some basic facts (as well as definitions) of noncommutative $L^{p}$-spaces associated with a von Neumann algebra which is not necessarily semifinite. Full details are found in $[3,8,12,14,15,30]$. Also we recall the theory of $\tau$-measurable operators arising from a semifinite von Neumann algebra. This subject was initiated by Segal [23], however, we will follow Nelson's elegant (and slightly different) treatment [20]. Details are found in $[\mathbf{1 8}, \mathbf{2 0}, \mathbf{2 1}, \mathbf{2 3}, \mathbf{2 6}$, 31].

In the first half of the section (and in main parts of the paper), we assume that $x$ is a (possibly type III) von Neumann algebra with a standard form ( $\mathscr{N}, \mathscr{H}, J, \mathscr{P P}^{\natural}$ ) in the sense of $[\mathbf{1}, \mathbf{9}]$. Also, throughout the paper we fix a unit cyclic (and separating) vector $\xi_{0}$ in $\mathcal{P}^{\natural}$ with the vector state $\phi_{0}=\omega_{\xi_{0}} \in \mathfrak{N}_{*}^{+}$, and denote the corresponding modular operator (on $\mathcal{H}$ ) by $\Delta$ [27].

We represent noncommutative $L^{p}$-spaces, $\left.L^{p}(\mathcal{O}) ; \phi_{0}\right), 0<p<\infty\left(L^{\infty}(\mathcal{O}) ; \phi_{0}\right)$ $=\mathcal{O}()$, as spaces of certain unbounded operators on $\mathcal{H}$ which are not affiliated with N (unless $\phi_{0}$ is tracial). More precisely, for each $\phi \in \mathcal{N}_{*}^{+}$with a unique implementing vector $\xi_{\phi}$ in $\mathcal{P}^{\natural}$, we consider the relative modular operator $\Delta_{\phi \phi_{0}}$ on $\mathscr{K}$ [7]. The positive selfadjoint operator $\Delta_{\phi \phi_{0}}$ is characterized as

$$
\left\{\begin{array}{l}
J \Delta_{\phi \phi_{0}}^{1 / 2} x \xi_{0}=x^{*} \xi_{\phi}, \quad x \in \mathfrak{N}, \\
\mathfrak{M} \xi_{0} \text { is a form core for } \Delta_{\phi \phi_{0}} .
\end{array}\right.
$$

Then $L^{p}\left(\mathscr{N} ; \phi_{0}\right), 0<p<\infty$, consists of all closed operators $a$ on $\mathscr{H}$ with the polar decomposition $a=u \Delta_{\phi \phi_{0}}^{1 / p}, u \in \mathfrak{R}, \phi \in \mathfrak{N}_{*}^{+}$. It is well known that one can freely add (strong sum), multiply (strong product), and take adjoints of elements in these $L^{p}$-spaces as if one deals with $\tau$-measurable operators (explained in the last half of the section). The positive part $L^{p}\left(\mathcal{O} ; \phi_{0}\right)_{+}$consists of all $\Delta_{\phi \phi_{0}}^{1 / p}, \phi \in \mathcal{O}_{*}^{+}$, and each element in $L^{p}\left(\mathcal{O} T ; \phi_{0}\right)$ can be written as a linear combination of four positive elements (in the usual way).

For each $a=u \Delta_{\phi \phi_{0}} \in L^{1}\left(\mathfrak{T} ; \phi_{0}\right)$, we set $\operatorname{tr}(a)="\left(a \xi_{0} \mid \xi_{0}\right) "$ in the form sense

$$
\left(=\left(\Delta_{\phi \phi_{0}}^{1 / 2} \xi_{0} \mid \Delta_{\phi \phi_{0}}^{1 / 2} u^{*} \xi_{0}\right)=\left(\xi_{\phi} \mid J u \xi_{\phi}\right)=\left(u \xi_{\phi} \mid \xi_{\phi}\right)\right)
$$

$$
=\phi(u) \text {. }
$$

As a consequence of the relative KMS condition, the (positive) linear functional $\mathrm{tr}$ on $L^{1}\left(\mathcal{O} ; \phi_{0}\right)$ enjoys the following tracial property:

$$
\operatorname{tr}(a b)=\operatorname{tr}(b a) ; \quad a \in L^{p}\left(\mathfrak{N} ; \phi_{0}\right), b \in L^{q}\left(\mathfrak{N} ; \phi_{0}\right) ; \quad 1 / p+1 / q=1 .
$$

For $p \geqslant 1, L^{p}\left(\mathscr{N} ; \phi_{0}\right)$ is a Banach space under the norm

$$
\|a\|_{p}=\operatorname{tr}\left(|a|^{p}\right)^{1 / p} \quad\left(=\phi(1)^{1 / p} \text { if } a=u \Delta_{\phi \phi_{0}}^{1 / p}\right),
$$


and is an M-bimodule in a natural way. (The module structure is defined by (strong) products of operators.)

Among these $L^{p}$-spaces, the $L^{1}$-space $\left.L^{1}(\Re) \phi_{0}\right)$ is isometrically isomorphic and order isomorphic to the predual $\Re_{*}$ via

$$
a=u \Delta_{\phi \phi_{0}} \rightarrow u \phi \quad(=\phi(u \cdot)),
$$

while the $L^{2}$-space $L^{2}\left(\mathscr{N} ; \phi_{0}\right)$ together with $L^{2}\left(\mathscr{N} ; \phi_{0}\right)_{+}$can be identified with $\left(\mathcal{K}, S^{\natural}\right)$ via

$$
a=u \Delta_{\phi \phi_{0}}^{1 / 2} \rightarrow a \xi_{0}=u \xi_{\phi}
$$

In particular, the inner product in $L^{2}\left(\Re ; \phi_{0}\right)$ is given by

$$
(a, b) \rightarrow \operatorname{tr}\left(a b^{*}\right)=\operatorname{tr}\left(b^{*} a\right) .
$$

(In fact, when $a=u \Delta_{\phi \phi_{0}}^{1 / 2}$ and $b=v \Delta_{\psi \phi_{0}}^{1 / 2}$, we have

$$
\begin{aligned}
\left(u \xi_{\phi} \mid v \xi_{\psi}\right) & =\left(u \Delta_{\phi \phi_{0}}^{1 / 2} \xi_{0} \mid v \Delta_{\psi \phi_{0}}^{1 / 2} \xi_{0}\right)=\left(\Delta_{\psi \phi_{0}}^{1 / 2} v^{*} u \Delta_{\phi \phi_{0}}^{1 / 2} \xi_{0} \mid \xi_{0}\right) \\
& \left.=\left(b^{*} a \xi_{0} \mid \xi_{0}\right)=\operatorname{tr}\left(b^{*} a\right) .\right)
\end{aligned}
$$

When $1 / p+1 / q=1$, the bilinear form

$$
(a, b) \in L^{p}\left(\Re ; \phi_{0}\right) \times L^{q}\left(\Re ; \phi_{0}\right) \rightarrow\langle a, b\rangle=\operatorname{tr}(a b) \in \mathbf{C}
$$

gives rise to the expected duality $L^{p}\left(\mathscr{T} ; \phi_{0}\right) \cong L^{q}\left(\mathfrak{T} ; \phi_{0}\right)^{*}, 1 \leqslant q<\infty$.

For each $1 \leqslant p \leqslant \infty$ with the conjugate exponent $q, L^{p}\left(\mathscr{N} ; \phi_{0}\right)$ may be continuously (due to Hölder's inequality) imbedded into $L^{1}\left(\mathscr{N} ; \phi_{0}\right) \cong \mathfrak{K}_{*}^{+}$via $a \rightarrow a \Delta^{1 / q}$. (Here, $\Delta=\Delta_{\phi_{0} \phi_{0}}$ is the usual modular operator fixed at the beginning of the section.) In particular, $\mathscr{N}=L^{\infty}\left(\mathfrak{K} ; \phi_{0}\right)$ is imbedded into $\mathfrak{K}_{*}$ via $x \rightarrow x \Delta(=x \phi)$ so that one obtains the pair $\left(\mathscr{N}, \mathscr{T}_{*}\right.$ ) of compatible Banach spaces in the sense of $[5,6]$.

Proposition 2.1 [15]. Under the above-mentioned imbedding, $L^{p}\left(\Re ; \phi_{0}\right)$ is exactly the complex interpolation space $C_{\theta=1 / p}\left(\mathfrak{K}, \Re_{*}\right)$ (with equal norms) in the sense of Calderón $[5,6]$.

In particular, $C_{1 / 2}\left(\mathfrak{N}, \mathscr{T}_{*}\right)=L^{2}\left(\mathfrak{N} ; \phi_{0}\right)=\mathscr{H}$, and the reiteration theorem for the complex interpolation method [6, Chapter 4] asserts that

$$
L^{p}\left(\mathscr{N} ; \phi_{0}\right)= \begin{cases}C_{2 / p}(\mathfrak{N}, \mathcal{H}), & 2<p<\infty, \\ C_{2 / p-1}\left(\mathcal{H}, \mathscr{N}_{*}\right), & 1<p<2,\end{cases}
$$

with equal norms (see $[\mathbf{1 5}, \S 4]$ for details). Thus, the result in the Appendix of [15] guarantees

Proposition 2.2 [15]. For each $1<p<\infty, L^{p}\left(\mathfrak{T} ; \phi_{0}\right)$ is a uniformly convex Banach space. Thus, due to (9) in $[17$, p. 365] and the duality, it is also a uniformly smooth Banach space.

We here recall that a Banach space $X$ is uniformly convex if the following condition is fulfilled: For each $\varepsilon>0$ there exists a $\delta=\delta_{\varepsilon}>0$ such that $\|x\|=\|y\|=1$ and $\left\|\frac{1}{2}(x+y)\right\|>1-\delta(x, y \in X)$ always imply $\|x-y\|<\varepsilon$. A very readable account on uniformly convex and smooth Banach spaces can be found in [17]. 
In the last half of the section, we assume that $\tau$ is a normal faithful semifinite trace ("gage") on a semifinite von Neumann algebra ơ acting on a Hilbert space $\mathfrak{h}$. Also, by an "operator" we will always mean a closed (and densely-defined) operator on $\mathcal{T}$ affiliated with $\mathscr{N}$.

An operator $a$ is said to be $\tau$-measurable if for any $\delta>0$ there exists a projection $e$ in $\mathscr{N}$ such that $e \mathfrak{K} \subseteq \mathscr{D}(a)$ and $\tau(1-e)<\delta[20]$.

Let $a=u|a|$ be the polar decomposition and $|a|=\int_{0}^{\infty} \lambda d e_{\lambda}$ the spectral decomposition. The following criterion is useful: $a$ is $\tau$-measurable if and only if there exists a positive $\lambda_{0}$ such that $\tau\left(1-e_{\lambda_{0}}\right)<\infty$ [20, p. 112]. As consequences of this fact: (i) if $\tau(1)<\infty$, then every operator is $\tau$-measurable; (ii) if an operator $a$ is bounded, that is, $a \in \mathfrak{N}$, then $a$ is $\tau$-measurable; (iii) if $a$ is $\tau$-measurable, then $\tau\left(1-e_{\lambda}\right) \searrow 0$ as $\lambda \nearrow \infty$. (Indeed, (iii) follows from $\tau\left(1-e_{\lambda_{0}}\right)<\infty, 1-e_{\lambda} \searrow 0$, and the normality of $\tau$.)

When $a$ and $b$ are $\tau$-measurable, then $a^{*}, b^{*}$ are $\tau$-measurable. Also, the (algebraic) sum $a+b$ and the (algebraic) product $a b$ are closable, and their closures $(a+b)^{-}$ (called the strong sum) and $(a b)^{-}$(called the strong product) are again $\tau$-measurable. Furthermore, under strong sum, strong product, and the adjoint operation, the set of all $\tau$-measurable operators forms a ${ }^{*}$-algebra $[\mathbf{2 0}, \mathbf{2 3}]$. Thus, in what follows, we will suppress a closure sign (without causing any ambiguity). Also, it is known [23, Theorem 5] that (iv) any symmetric $\tau$-measurable operator is selfadjoint.

We now introduce a topology on the set of all $\tau$-measurable operators (see [20, $\S 2])$. For $\varepsilon, \delta>0$, we set

$$
\begin{aligned}
& \varrho(\varepsilon, \delta)=\{a ; a \text { is a } \tau \text {-measurable operator admitting a projection } \\
& \qquad e \in \mathfrak{N} \text {, with }\|a e\| \leqslant \varepsilon \text { and } \tau(1-e) \leqslant \delta\} .
\end{aligned}
$$

A vector topology whose fundamental system of neighborhoods around 0 is $\{\hat{C}(\varepsilon, \delta)\}_{\varepsilon, \delta>0}$ will be referred to as the measure topology. It is known [20] that (v) the set of all $\tau$-measurable operators equipped with the measure topology forms a Hausdorff topological *-algebra. (It is complete, but not locally convex.) We note that (iii) implies (iii)': if $a$ is $\tau$-measurable, $a_{n}=u \int_{0}^{n} \lambda d e_{\lambda}$ ( $\in \mathcal{O M}$ ) tends to $a$ (as $n \rightarrow \infty)$ in measure.

Now, we describe noncommutative $L^{p}$-spaces $\left.L^{p}(\mathfrak{O}) ; \tau\right), 0<p \leqslant \infty$. Although $\tau$ can be a trace with $\tau(1)=\infty$, we assume that $\tau$ is a tracial state ("probability gage") in what follows. (In fact, in $\$ \S 3$ and 5 , we will use just noncommutative $L^{p}$-spaces arising from a tracial state.)

In the literature [18, 20], of course, $L^{p}(\mathfrak{T} ; \tau), 1 \leqslant p<\infty$, is defined as the space consisting of all operators $a$ (recall (i)) satisfying $\tau\left(|a|^{p}\right)<\infty$. It is a Banach space under the norm $\|a\|_{p}=\tau\left(|a|^{p}\right)^{1 / p}$ and satisfies all expected properties such as duality. We point out that (when $\mathscr{N}$ is standardly represented) $L^{p}\left(\mathfrak{N} ; \phi_{0}=\tau\right)$ in the sense of the first half of the section is exactly $L^{p}(\Re ; \tau)$ just defined. In fact, when $\phi_{0}=\tau$ (that is, $\xi_{0}=\xi_{\tau}$ is a unit trace vector), a relative modular operator $\Delta_{\phi \tau}=h_{\phi}$ is affiliated with $\mathscr{N}$ and is the Radon-Nikodym derivative $d \phi / d \tau$ in the classical sense. Furthermore, norms are computed by

$$
\operatorname{tr}\left(\Delta_{\phi \tau}\right)=\left(\Delta_{\phi \tau} \xi_{\tau} \mid \xi_{\tau}\right)=\tau\left(h_{\phi}\right)
$$


We note (vi): If a sequence $\left\{a_{n}\right\}$ in $L^{p}(\mathscr{N} ; \tau), 0<p<\infty$, tends to $a$ in the $L^{p}$-norm, then $a_{n}$ tends to $a$ in measure. In fact, if $b=u|b|$ and $|b|=\int_{0}^{\infty} \lambda d e_{\lambda}$ (the spectral decomposition), then

$$
\|b\|_{p}^{p}=\tau\left(|b|^{p}\right)=\int_{0}^{\infty} \lambda^{p} d \tau\left(e_{\lambda}\right) \geqslant \int_{\varepsilon}^{\infty} \lambda^{p} d \tau\left(e_{\lambda}\right) \geqslant \varepsilon^{p} \tau\left(1-e_{\varepsilon}\right) \quad(\varepsilon>0) .
$$

Thus, for any preassigned $\varepsilon, \delta>0,\|b\|_{p} \leqslant \varepsilon \delta^{1 / p}$ implies $\tau\left(1-e_{\varepsilon}\right) \leqslant \delta$ so that $\left\{b \in L^{p}(\mathscr{R} ; \tau):\|b\|_{p} \leqslant \varepsilon \delta^{1 / p}\right\}$ is included in $\Theta(\varepsilon, \delta)$.

For bounded selfadjoint operators $a, b$, it is well known that $a b=b a$ if and only if $a$ and $b$ commute strongly, that is, $e^{i \alpha a} e^{i \beta b}=e^{i \beta b} e^{i \alpha a}, \alpha, \beta \in \mathbf{R}$, or, equivalently, all spectral projections of $a$ and $b$ mutually commute. However, for general unbounded selfadjoint operators, the situation is much worse. Indeed, as the famous counterexample of Nelson [22, p. 273] indicates, all kinds of pathological phenomena occur. We, however, show that for special unbounded selfadjoint operators appearing in $\$ 2$ no pathological phenomenon occurs.

LEMMA 2.3. Let $a, b$ be $\tau$-measurable selfadjoint operators. (Here, Th need not be finite.) Then $a b=b a$ if and only if $a$ and $b$ commute strongly.

Proof. If $[a, b]=0$, we get $[P(a), Q(b)]=0$ for any two polynomials $P, Q$. Let $f, g$ be two bounded continuous functions on $\mathbf{R}$, and $P_{n}, Q_{n}$ sequences of polynomials converging to $f$ and $g$ uniformly on compact subsets of $\mathbf{R}$. For $\varepsilon, \delta>0$, one can choose $t_{0}>0$ such that the spectral projection of $a$ corresponding to the set $\{t \in \mathbf{R}$; $\left.|t|>t_{0}\right\}$ has trace $\leqslant \delta$, and then choose $n_{0} \in \mathbf{N}$ such that

$$
\left|P_{n}(t)-f(t)\right| \leqslant \varepsilon \quad \text { for } t \in\left[-t_{0}, t_{0}\right], n \geqslant n_{0} .
$$

Then $P_{n}(a)-f(a) \in \mathcal{O}(\varepsilon, \delta), n \geqslant n_{0}$, and $P_{n}(a)$ tends to $f(a)$ in measure. Similarly, $Q_{n}(b)$ tends to $g(b)$ in measure. Since the set of all $\tau$-measurable operators form a topological algebra, we get

$$
[f(a), g(b)]=\lim _{n \rightarrow \infty}\left[P_{n}(a), Q_{n}(b)\right]=0 .
$$

Taking $f(t)=e^{i \alpha t}$ and $g(t)=e^{i \beta t}$, one concludes that $a$ and $b$ commute strongly. Q.E.D.

LEMMA 2.4. Let $\phi, \psi \in \mathfrak{M}_{*}^{+}$and $\alpha, \beta>0$. (Here, $\Re$ need not be semifinite.) Then $\Delta_{\phi \phi_{0}}^{\alpha} \Delta_{\psi \phi_{0}}^{\beta}=\Delta_{\psi \phi_{0}}^{\beta} \Delta_{\phi \phi_{0}}^{\alpha}$ if and only if $\Delta_{\phi \phi_{0}}$ and $\Delta_{\psi \phi_{0}}$ commute strongly.

Proof. We consider the crossed product $\Re \rtimes_{\sigma} \mathbf{R}$ (relative to the modular automorphism group $\left.\sigma_{t}=\operatorname{Ad} \Delta^{i t}, t \in \mathbf{R}\right)$ acting on $\mathcal{H} \otimes L^{2}(\mathbf{R} ; d t)$ generated by $x \otimes 1, x \in \mathfrak{M}$, and $\Delta^{i t} \otimes L^{i t}, t \in \mathbf{R}$. Here, $L$ is the exponential of the generator of the regular representation of $\mathbf{R}$. Then the crossed product is semifinite, and it is known $[12, \S 1]$ that $h_{\phi}=\Delta_{\phi \phi_{0}} \otimes L$ is $\tau$-measurable with respect to the canonical trace $\tau$ on the crossed product. (See [22, VIII-10] for tensor products of unbounded operators.) 
We thus have the following bi-implications:

$$
\begin{aligned}
{\left[\Delta_{\phi \phi_{0}}^{\alpha}, \Delta_{\psi \phi_{0}}^{\beta}\right]=0 } & \Leftrightarrow\left[h_{\phi}^{\alpha}, h_{\psi}^{\beta}\right]=0 \\
& \Leftrightarrow h_{\phi} \text { and } h_{\psi} \text { commute strongly (Lemma 2.3) } \\
& \Leftrightarrow \Delta_{\phi \phi_{0}}^{i t} \otimes L^{i t} \text { and } \Delta_{\psi \phi_{0}}^{i s} \otimes L^{i s} \text { comute } \\
& \Leftrightarrow \Delta_{\phi \phi_{0}}^{i t} \text { and } \Delta_{\psi \phi_{0}}^{i s} \text { commute. Q.E.D. }
\end{aligned}
$$

3. Technical lemmas. In this section we collect some technical lemmas. Among other results, in the last half, certain dominated convergence theorems for a probability gage will be obtained (Proposition 3.7 and Corollary 3.8).

Lemma 3.1. If $t \in \mathbf{R} \rightarrow h(t) \in L^{p}\left(\mathfrak{T} ; \phi_{0}\right)_{+}, 1<p<\infty$, is differentiable (with

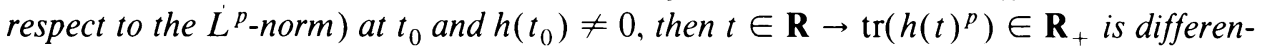
tiable at $t_{0}$ and its derivative is

$$
\left.p \operatorname{tr}\left(h\left(t_{0}\right)\right)^{p-1} \frac{d}{d t}\right|_{t=t_{0}} h\left(t_{0}\right) .
$$

Two remarks are in order before its proof. Firstly, the assumption asserts that, if one sets $b_{\varepsilon}=h\left(t_{0}+\varepsilon\right)-h\left(t_{0}\right)$ for a small $\varepsilon \neq 0 \in \mathbf{R}, \varepsilon^{-1} b_{\varepsilon}$ tends to $\left.(d / d t)\right|_{t=t_{0}} h(t)$ in norm as $\varepsilon \rightarrow 0$. Notice that $\varepsilon^{-1} b_{\varepsilon}$ and $\left.(d / d t)\right|_{t=t_{0}} h(t)$ belong to $L^{p}\left(\text { O } T ; \phi_{0}\right)_{s a}=$ $\left.\left\{a \in L^{p}(\Re) ; \phi_{0}\right) ; a^{*}=a\right\}$. Secondly, it is easily observed that the real Banach spaces $\left.L^{p}(\mathcal{O}) ; \phi_{0}\right)_{s a}$ and $\left.L^{q}(\mathcal{O}) ; \phi_{0}\right)_{s a}$ are uniformly smooth and convex and the dual spaces of each other (see $\$ 2$ ). More precisely, the duality here is given by restricting the bilinear form mentioned in $\$ 2$ to $\left.L^{p}(\Re) ; \phi_{0}\right)_{s a} \times L^{q}\left(\Omega \Re ; \phi_{0}\right)_{s a}$.

PROOF. It is known that the norm function $\left.a \in L^{p}(\mathcal{O}) ; \phi_{0}\right)_{s a} \rightarrow\|a\|_{p} \in \mathbf{R}_{+}$is strongly differentiable (Fréchet differentiable) except at 0 due to the uniform smoothness of $L^{p}\left(\mathcal{O K} ; \phi_{0}\right)_{s a}[17,(6), \mathrm{p} .364]$. At first we show that its strong derivative at $a(\geqslant 0, \neq 0)$ is $\|a\|_{p}^{1-p} a^{p-1}$ (in $L^{q}\left(\mathcal{O} ; \phi_{0}\right)_{s a} \cong L^{p}\left(\mathcal{O} ; \phi_{0}\right)_{s a}^{*}$ ). The space $L^{p}\left(\mathcal{O}, \phi_{0}\right)_{s a}$ being strictly smooth [17, p. 353], there exists only one supporting hyperplane for the ball in $L^{p}\left(\mathcal{N} ; \phi_{0}\right)_{s a}$ of radius $\|a\|_{p}$ containing $a$. Furthermore, $a$ is the only point where this supporting hyperplane and the boundary of the ball meet. However, this supporting hyperplane is

$$
\left\{b \in L^{p}\left(\mathscr{N} ; \phi_{0}\right)_{s a}: \operatorname{tr}\left(\|a\|_{p}^{1-p} a^{p-1} b\right)=\|a\|_{p}\right\}
$$

because of

$$
\begin{aligned}
& \operatorname{tr}\left(\|a\|_{p}^{1-p} a^{p-1} a\right)=\|a\|_{p}, \\
& \operatorname{tr}\left(\|a\|_{p}^{1-p} a^{p-1} b\right) \leqslant\|a\|_{p} \quad \text { whenever } b^{*}=b,\|b\|_{p} \leqslant\|a\|_{p} .
\end{aligned}
$$

Consequently, the strong derivative of the norm function at $a$ is $\operatorname{tr}\left(\|a\|^{1-p} a^{p-1} \cdot\right) \cong$ $\|a\|^{1-p} a^{p-1}$. (See [17, (11), p. 349 and (4), p. 364].)

Thanks to a chain rule, the function $a \in L^{p}\left(\mathscr{T} ; \phi_{0}\right)_{s a} \rightarrow\|a\|_{p}^{p} \in \mathbf{R}_{+}$is strongly differentiable except at 0 , and its strong derivative at $h\left(t_{0}\right)$ is

$$
\left(p\left\|h\left(t_{0}\right)\right\|_{p}^{p-1}\right)\left(\left\|h\left(t_{0}\right)\right\|^{1-p} h\left(t_{0}\right)^{p-1}\right)=p h\left(t_{0}\right)^{p-1} .
$$


Using $\varepsilon, \delta_{\varepsilon}$ introduced before the proof, we compute

$$
\begin{aligned}
\varepsilon^{-1}\left\{\operatorname{tr}\left(h\left(t_{0}+\varepsilon\right)^{p}\right)-\operatorname{tr}\left(h\left(t_{0}\right)^{p}\right)\right\}=\varepsilon^{-1}\left\{\left\|h\left(t_{0}\right)+b_{f}\right\|_{p}^{p}-\left\|h\left(t_{0}\right)\right\|_{p}^{p}\right\} \\
=\varepsilon^{-1}\left\{\left\|h\left(t_{0}\right)\right\|_{p}^{p}+\operatorname{tr}\left(p h\left(t_{0}\right)^{p-1} b_{\varepsilon}\right)+\delta_{\varepsilon}-\left\|h\left(t_{0}\right)\right\|_{p}^{p}\right\} \\
=\operatorname{tr}\left(p h\left(t_{0}\right)^{p-1} \varepsilon^{-1} b_{\varepsilon}\right)+\varepsilon^{-1} \delta_{\varepsilon} .
\end{aligned}
$$

Here,

$$
\delta_{\varepsilon}=\left\|h\left(t_{0}\right)+b_{\varepsilon}\right\|_{p}^{p}-\left\|h\left(t_{0}\right)\right\|_{p}^{p}-\operatorname{tr}\left(p h\left(t_{0}\right)^{p-1} b_{\varepsilon}\right),
$$

and $\delta_{\varepsilon}\left\|b_{\varepsilon}\right\|_{p}^{-1}$ tends to 0 as $\left\|b_{\varepsilon}\right\|_{p}$ tends to 0 (by what we showed in the first half of the proof). Since $\varepsilon^{-1} b_{\varepsilon}$ tends to $\left.(d / d t)\right|_{t=t_{0}} h(t)$ as $\varepsilon \rightarrow 0$, the results now follow from the above computation. Q.E.D.

The next result was proved in [29] for traces on semifinite algebras. For the sake of completeness we present a proof in our set-up.

Lemma 3.2 [29, Lemma 2(i), (iii)]. Let $a, b$ be elements in $\left.L^{p}(\mathscr{O}) ; \phi_{0}\right)_{+}$and $a \leqslant b$ (as operators).

(i) $\operatorname{tr}\left(a^{p}\right) \leqslant \operatorname{tr}\left(b^{p}\right), 0<p<\infty$,

(ii) $\operatorname{tr}\left(a^{p}\right) \leqslant \operatorname{tr}\left(\sqrt{a} b^{p-1} \sqrt{a}\right)=\operatorname{tr}\left(b^{p-1} a\right), 1<p<\infty$.

Proof [29]. (i) At first we prove it for $p=2^{n}, n=0,1,2, \ldots$, by induction on $n$. For $n=0$ the result is obvious, hence we assume it for $p=2^{n-1}$. Since $\sqrt{a} a \sqrt{a}$ $\leqslant \sqrt{a} b \sqrt{a}, \sqrt{b} a \sqrt{b} \leqslant \sqrt{b} b \sqrt{b}$, we estimate

$$
\begin{aligned}
\operatorname{tr}\left(a^{2 n}\right) & =\operatorname{tr}\left((\sqrt{a} a \sqrt{a})^{2 n-1}\right) \leqslant \operatorname{tr}\left((\sqrt{a} b \sqrt{a})^{2 n-1}\right)=\operatorname{tr}\left((\sqrt{b} a \sqrt{b})^{2 n-1}\right) \\
& \leqslant \operatorname{tr}\left((\sqrt{b} b \sqrt{b})^{2 n-1}\right)=\operatorname{tr}\left(b^{2 n}\right) .
\end{aligned}
$$

Here, in the third expression on the right-hand side, the tracial property was used, and the induction hypothesis was also used twice. For a general $p$ we write $p=2^{n} p^{\prime}$ with $0<p^{\prime} \leqslant 1$. The operator monotonicity of $t^{p^{\prime}}, t \geqslant 0$ (hence $a^{p^{\prime}} \leqslant b^{p^{\prime}}$ ), and the first half of the proof imply

$$
\operatorname{tr}\left(a^{p}\right)=\operatorname{tr}\left(\left(a^{p^{\prime}}\right)^{2 n}\right) \leqslant \operatorname{tr}\left(\left(b^{p^{\prime}}\right)^{2 n}\right)=\operatorname{tr}\left(b^{p}\right) .
$$

(ii) Actually, by induction, we will show that $\operatorname{tr}\left(a^{m p}\right) \leqslant \operatorname{tr}\left(\left(\sqrt{a} b^{p-1} \sqrt{a}\right)^{m}\right)$ for $1<p<\infty, 0<m<\infty, a, b \in L^{m p}\left(\mathscr{N} ; \phi_{0}\right)_{+}$with $a \leqslant b$. If $p=1+\alpha, 0<\alpha \leqslant 1$, the operator monotonicity of $t^{\alpha}, t \geqslant 0$, shows

$$
a^{p}=\sqrt{a} a^{\alpha} \sqrt{a} \leqslant \sqrt{a} b^{\alpha} \sqrt{a}=\sqrt{a} b^{p-1} \sqrt{a} .
$$

Hence, the result follows from (i). We now assume that $p=n+\alpha, n \in \mathbf{N}_{+}, n \geqslant 2$, $0<\alpha \leqslant 1$, and that the result is true for $p$ of the form $l+\beta, l \in\{1,2, \ldots, n-1\}$, $0<\beta \leqslant 1$. We observe that

$$
\begin{aligned}
\left(\sqrt{a} b^{p / 2-1} \sqrt{a}\right)^{2} & =\sqrt{a} b^{p / 2-1} a b^{p / 2-1} \sqrt{a} \\
& \leqslant \sqrt{a} b^{p / 2-1} b b^{p / 2-1} \sqrt{a}=\sqrt{a} b^{p-1} \sqrt{a} .
\end{aligned}
$$


Since $p / 2-1=l^{\prime}+\beta^{\prime}$ with $l^{\prime} \in\{1,2, \ldots, n-1\}$ and $0<\beta^{\prime} \leqslant 1$, we get

$$
\begin{aligned}
\operatorname{tr}\left(\left(\sqrt{a} b^{p-1} \sqrt{a}\right)^{m}\right) & \geqslant \operatorname{tr}\left(\left(\sqrt{a} b^{p / 2-1} \sqrt{a}\right)^{2 m}\right) \quad(\text { the above estimate and (i) }) \\
& \geqslant \operatorname{tr}\left(\left(a^{p / 2}\right)^{2 m}\right) \quad \text { (induction hypothesis) } \\
& =\operatorname{tr}\left(a^{p m}\right) . \quad \text { Q.E.D. }
\end{aligned}
$$

LeMma 3.3. For $a, b$ in $L^{p}\left(\mathfrak{N} ; \phi_{0}\right)_{+}, 1<p<\infty$, we have

$$
\operatorname{tr}\left(a^{p}+b^{p}\right) \leqslant \operatorname{tr}\left((a+b)^{p}\right) .
$$

Proof. We may and do assume $a \neq 0$; for $t \geqslant 0$ consider the continuous function

$$
g(t)=\operatorname{tr}\left((a+t b)^{p}\right)-\operatorname{tr}\left(a^{p}+t^{p} b^{p}\right) .
$$

For $t>0$, Lemmas 3.1 and 3.2(ii) yield

$$
\begin{aligned}
g^{\prime}(t) & =p \operatorname{tr}\left((a+t b)^{p-1}\right)-p t^{p-1} \operatorname{tr}\left(b^{p}\right) \\
& =p t^{p-1}\left\{\operatorname{tr}\left(\left(b+t^{-1} a\right)^{p-1} b\right)-\operatorname{tr}\left(b^{p}\right)\right\} \geqslant 0 .
\end{aligned}
$$

We thus get

$$
g(1)=\operatorname{tr}\left((a+b)^{p}\right)-\operatorname{tr}\left(a^{p}+b^{p}\right) \geqslant g(0)=0 . \quad \text { Q.E.D. }
$$

Lemma 3.4. For each $1<p<\infty$ and positive $a$, $b$ in $L^{1}\left(\mathfrak{M} ; \phi_{0}\right) \cong \mathfrak{M}_{*}$, we have

$$
\left\|a^{1 / p}-b^{1 / p}\right\|_{p} \leqslant 2^{1-1 / p}\|a-b\|_{1}^{1 / p} \text {. }
$$

In particular, the map $a \in L^{1}\left(\mathscr{N} ; \phi_{0}\right)_{+} \rightarrow a^{1 / p} \in L^{p}\left(\mathfrak{R} ; \phi_{0}\right)_{+}$is norm-continuous.

Proof. At first we assume $a \geqslant b \geqslant 0$ and set $c=a^{1 / p}-b^{1 / p}, d=b^{1 / p}$. Owing to the operator monotonicity of $t^{1 / p}, t \geqslant 0, c$ (and $d$ ) are in $\left.L^{p}(:) ; \phi_{0}\right)_{+}$. We compute

$$
\begin{aligned}
\|a-b\|_{1}-\left\|a^{1 / p}-b^{1 / p}\right\|_{p}^{p} & =\operatorname{tr}(a-b)-\operatorname{tr}\left(c^{p}\right)=\operatorname{tr}(a)-\left\{\operatorname{tr}(b)+\operatorname{tr}\left(c^{p}\right)\right\} \\
& =\operatorname{tr}\left((c+d)^{p}\right)-\operatorname{tr}\left(d^{p}+c^{p}\right) \geqslant 0
\end{aligned}
$$

because of Lemma 3.3. We have thus proved

$$
\left\|a^{1 / p}-b^{1 / p}\right\|_{p} \leqslant\|a-b\|_{1}^{1 / p} \quad \text { if } a \geqslant b \geqslant 0 .
$$

For general $a, b \geqslant 0$, considering the Jordan decomposition $a-b=(a-b)_{+}$ $-(a-b)_{-}$, we estimate

$$
\begin{aligned}
\left\|a^{1 / p}-b^{1 / p}\right\|_{p} \leqslant & \left\|a^{1 / p}-\left(b+(a-b)_{+}\right)^{1 / p}\right\|_{p}+\left\|\left(b+(a-b)_{+}\right)^{1 / p}-b^{1 / p}\right\|_{p} \\
= & \left\|\left(b+(a-b)_{+}\right)^{1 / p}-\left(b+(a-b)_{+}-(a-b)_{-}\right)^{1 / p}\right\|_{p} \\
& +\left\|\left(b+(a-b)_{+}\right)^{1 / p}-b^{1 / p}\right\|_{p} \\
\leqslant & \left\|(a-b)_{-}\right\|_{1}^{1 / p}+\left\|(a-b)_{+}\right\|_{1}^{1 / p} \quad \text { (by the first half of the proof) } \\
\leqslant & 2^{1-1 / p}\left\{\left\|(a-b)_{+}\right\|_{1}+\left\|(a-b)_{-}\right\|_{1}\right\}^{1 / p} .
\end{aligned}
$$


Here, the last inequality is just the concavity of $t^{1 / p}, t \geqslant 0$. However, since $(a-b)$. sum up to $|a-b|$, we get

$$
\begin{aligned}
\left\|(a-b)_{+}\right\|_{1}+\left\|(a-b)_{-}\right\|_{1} & =\operatorname{tr}\left((a-b)_{+}+(a-b)_{-}\right) \\
& =\operatorname{tr}(|a-b|)=\|a-b\|_{1} . \quad \text { Q.E.D. }
\end{aligned}
$$

The next result is part of the standard result, but we state and prove it because it will be repeatedly used.

LEMMA 3.5. Let $\left\{x_{n}\right\}$ be a sequence in a uniformly convex Banach space $X$ and $x \in X$ such that $\lim _{n \rightarrow \infty}\left\|x_{n}\right\|=\|x\|$. Then $x_{n}$ tends to $x$ in norm if there exists an element $f$ in $X^{*}$, the dual of $X$, such that $f(x)=\|f\|_{X^{*}}\|x\|$ and $\lim _{n \rightarrow \infty} f\left(x_{n}\right)=f(x)$. (In particular, if $x_{n}$ tends to $x$ weakly, the condition is fulfilled due to the Hahn-Banach theorem.)

Proof. When $x=0$ there is nothing to prove. Thus we assume $x \neq 0$. Hence $x_{n} \neq 0$ for $n$ large enough (or even for all $n$ ). After normalization (it does not change a convergence property because $\left.\lim _{n \rightarrow \infty}\left\|x_{n}\right\|=\|x\|\right)$ we may and do assume $\left\|x_{n}\right\|=$ $\|x\|=1$ and $\|f\|_{X^{*}}=f(x)=1$. We then have

$$
(1 \geqslant)\left\|\frac{1}{2}\left(x+x_{n}\right)\right\| \geqslant f\left(\frac{1}{2}\left(x+x_{n}\right)\right) \rightarrow f(x)=1
$$

as $n \rightarrow \infty$. Thus, the result follows from uniform convexity. Q.E.D.

In the rest of the section, we assume $\Re$ is finite and $\tau$ is a faithful tracial state on it, and let $L^{p}(\Re ; \tau), 0<p \leqslant \infty$, be the corresponding $L^{p}$-space explained in the second half of $\S 2$.

We recall Hansen's inequality [11]: $x^{*} g(a) x \leqslant g\left(x^{*} a x\right)$ for a contraction $x$ $(\|x\| \leqslant 1)$, a positive bounded operator $a$, and an operator monotone function $g(\lambda)$ on $[0, \infty)$. For a contraction $x$ in $\Re$ and a positive closed (hence selfadjoint and $\tau$-measurable, (i), (iv) in $\$ 2$ ) operator $a$ affiliated with $\mathfrak{N}$, the operator inequality remains valid. Indeed, due to (iii)' in $\$ 2$, one can pick up a sequence $\left\{a_{n}\right\}$ in $\Re_{+}$ converging increasingly to $a$ in the measure topology. Since $x^{*} g\left(a_{n}\right) x \leqslant g\left(x^{*} a_{n} x\right) \leqslant$ $g\left(x^{*} a x\right)$, letting $n \uparrow \infty$, we obtain $x^{*} g(a) x \leqslant g\left(x^{*} a x\right)$.

LEMMA 3.6. Let a be an element in $L^{p}(\Re ; \tau){ }_{+}$and $x$ a contraction in $\Re$.

(i) If $0<p<1$, then $\tau\left(x^{*} a^{p} x\right) \leqslant \tau\left(\left(x^{*} a x\right)^{p}\right)$.

(ii) If $1<p<\infty$, then $\tau\left(x^{*} a^{p} x\right) \geqslant \tau\left(\left(x^{*} a x\right)^{p}\right)$.

Proof. (i) The operator monotonicity of $t^{p}, t \geqslant 0$, and Hansen's inequality imply $x^{*} a^{p} x \leqslant\left(x^{*} a x\right)^{p}$ from which the result follows.

(ii) Since $0<1 / p<1$, we now have

$$
x^{*} a x=x^{*}\left(a^{p}\right)^{1 / p} x \leqslant\left(x^{*} a^{p} x\right)^{1 / p} .
$$

Thus, the result follows from Lemma 3.2(i) (or rather [29, Lemma 2(ii)]). Q.E.D.

The next result (and its corollary) can be regarded as a dominated convergence theorem for a probability gage. Although we will use just a special case $(p=2)$ of this result, we state and prove it in full generality. 
Proposition 3.7. Let $\left\{a_{n}\right\}$ be a sequence in $L^{p}(\mathfrak{T} ; \tau), 2 \leqslant p<\infty$, and $a \in$ $L^{p}(\mathfrak{T} ; \tau)$. If $a_{n}$ tends to $a$ in measure and $\left|a_{n}\right|^{p}$ tends to $|a|^{p}$ in the $L^{1}$-norm, then $a_{n}$ tends to $a$ in the $L^{p}$-norm.

Proof. Assume $\left|a_{n}\right|^{p}$ (resp. $|a|^{p}$ ) corresponds to $\phi\left(\right.$ resp. $\phi_{n}$ ) in $\Re_{*}$. Since $\phi_{n}$ tends to $\phi$ in the predual norm, for each $\varepsilon>0$ we get $\left\|\phi_{n}-\phi_{m}\right\|<\varepsilon$ if $n, m \geqslant N=N_{\varepsilon}$. We can obviously choose a positive $x(\neq 0)$ in $\mathfrak{M}$ satisfying $\left\|\phi_{N}-\tau x\right\|<\varepsilon$. Fixing this $x$, we set $\delta\left(=\delta_{\varepsilon}\right)=\varepsilon /\|x\|$.

For each projection $e \in \mathfrak{M}$ with $\tau(e) \leqslant \delta$, we have $(0 \leqslant) \phi_{n}(e)<3 \varepsilon$ (hence $\phi(e) \leqslant 3 \varepsilon$ ) whenever $n \geqslant N$. In fact we estimate

$$
\begin{aligned}
\phi_{n}(e) & \leqslant\left|\left(\phi_{n}-\phi_{N}\right)(e)\right|+\left|\left(\phi_{N}-\tau x\right)(e)\right|+\tau(x e) \\
& <\varepsilon\|e\|+\varepsilon\|e\|+\|x\| \tau(e) \leqslant 3 \varepsilon .
\end{aligned}
$$

Since $1 \leqslant p / 2<\infty$ (for this $e, \tau(e) \leqslant \delta$ ), we get

$$
\begin{aligned}
\left\|a_{n} e\right\|_{p}^{p} & \left.=\tau\left(\left|a_{n} e\right|^{p}\right)=\left(\left(e a_{n}^{*} a_{n} e\right)^{p / 2}\right)=\tau\left(\left(e\left|a_{n}\right|^{2} e\right)\right)^{p / 2}\right) \\
& \leqslant\left(e\left(\left|a_{n}\right|^{2}\right)^{p / 2} e\right) \quad(\text { Lemma 3.6(ii) }) \\
& =\left(\left|a_{n}\right|^{p} e\right)=\phi_{n}(e)<3 \varepsilon .
\end{aligned}
$$

The same arguments also show $\|a e\|_{p}^{p} \leqslant 3 \varepsilon$.

The sequence $\left\{a_{n}\right\}$ tending to $a$ in measure, for $n \geqslant M=M_{\varepsilon}$ (and for the above $\varepsilon$ and $\left.\delta_{\varepsilon}\right)$ there exists a projection $e$ (depending on $\left.n\right)$ such that $\left\|\left(a_{n}-a\right)(1-e)\right\| \leqslant \varepsilon$ and $\tau(e) \leqslant \delta$ (see $\S 2$ ). The "gage" $\tau$ being probability, the uniform norm \| $\|$ majorizes the $L^{p}$-norm \|\|$_{p}$. Thus, whenever $n \geqslant M$, the following estimates are valid:

$$
\begin{aligned}
\left\|a_{n}-a\right\|_{p} & \leqslant\left\|\left(a_{n}-a\right)(1-e)\right\|_{p}+\left\|\left(a_{n}-a\right) e\right\|_{p} \\
& \leqslant\left\|\left(a_{n}-a\right)(1-e)\right\|_{p}+\left\|a_{n} e\right\|_{p}+\|a e\|_{p} \leqslant \varepsilon+\left\|a_{n} e\right\|_{p}+\|a e\|_{p} .
\end{aligned}
$$

Hence, if $n \geqslant \operatorname{Max}(N, M)$, then

$$
\left\|a_{n}-a\right\|_{p}<\varepsilon+(3 \varepsilon)^{1 / p}+(3 \varepsilon)^{1 / p} \text {. Q.E.D. }
$$

It is known ([21, Proposition 1] and (iv) in §2) that, for $b_{n}, b$ in $L^{1}(\mathscr{T} ; \tau)_{+}$, $\lim _{n \rightarrow \infty}\left\|b_{n}-b\right\|_{1}=0$ if and only if $\lim _{n \rightarrow \infty}\left\|b_{n}\right\|_{1}=\|b\|_{1}$ and $b_{n}$ tends to $b$ in measure. Based on this we show

Corollary 3.8. Let $\left\{a_{n}\right\}$ be a sequence in $L^{p}(\mathscr{N} ; \tau), 2 \leqslant p<\infty$, and $a \in$ $L^{p}(\mathfrak{O} ; \tau)$. The sequence tends to $a$ in the $L^{p}$-norm if and only if $a_{n}$ tends to $a$ in measure and $\lim _{n \rightarrow \infty}\left\|a_{n}\right\|_{p}=\|a\|_{p}$.

Proof. If $\lim _{n \rightarrow \infty}\left\|a_{n}-a\right\|_{p}=0$, then $\lim _{n \rightarrow \infty}\left\|a_{n}\right\|_{p}=\|a\|_{p}$ and $a_{n}$ tends to $a$ in measure ((iv) in $§ 2)$.

Conversely, assume these two conditions are fulfilled. Firstly, $\left\|\left|a_{n}\right|^{p}\right\|_{1}=\tau\left(\left|a_{n}\right|^{p}\right)$ $=\left\|a_{n}\right\|_{p}^{p}$ tends to $\left\||a|^{p}\right\|_{1}=\|a\|_{p}^{p}$. Secondly, $\tau$ being probability, the map: $b \rightarrow$ $\left(b^{*} b\right)^{p / 2}$ is continuous with respect to the measure topology [21, Theorem 2.1]. 
Therefore, $\left|a_{n}\right|^{p}$ tends to $|a|^{p}$ in measure, hence in the $L^{1}$-norm, thanks to the remark right before the corollary. It follows from Proposition 3.7 that $\left|a_{n}\right|$ tends to $a$ in the $L^{p}$-norm. Q.E.D.

Theorem 3.1 in [21] asserts that Corollary 3.8 is also valid for $p=1$.

4. Continuity of the "absolute value part" map. We prove the norm-continuity of the "absolute value part" map from our $L^{p}$-space onto its positive part.

Lemma 4.1. We assume $1 / p+1 / q=1,1<p<\infty$. For each $a \in L^{p}\left(\Re ; \phi_{0}\right)$ with the polar decomposition $a=u|a|$, we set $\pi(a)=|a|^{p / q} u^{*}(\pi(a)=0$ if $a=0)$ so $\pi(a)$ belongs to $L^{q}\left(\mathfrak{N} ; \phi_{0}\right)$. Then the map $\pi: L^{p}\left(\Re ; \phi_{0}\right) \rightarrow L^{q}\left(\Re ; \phi_{0}\right)$ is norm-continuous.

Proof. For $a=u|a|, u^{*} u$ is the projection $(\in \mathscr{N})$ onto the support of $|a|$ so $|a|^{p / q} u^{*} u=|a|^{p / q}$. We thus compute

$$
\left\||a|^{p / q} u^{*}\right\|_{q} \leqslant\left\||a|^{p / q}\right\|_{q}=\left\||a|^{p / q} u^{*} u\right\|_{q} \leqslant\left\||a|^{p / q} u^{*}\right\|_{q}
$$

that is,

$$
\|\pi(a)\|_{q}=\left\||a|^{p / q}\right\|_{q}=\operatorname{tr}\left(|a|^{p}\right)^{1 / q}=\|a\|_{p}^{p / q} .
$$

We now assume $\left\{a_{n}\right\}$ in $L^{p}\left(\mathscr{N} ; \phi_{0}\right)$ tends to $a \neq 0$ in norm. Thanks to the above equality, as in the proof of Lemma 3.5, we may and do assume $\left\|a_{n}\right\|_{p}=\|a\|_{p}=1$ (hence $\left.\|\pi(a)\|_{q}=\left\|\pi\left(a_{n}\right)\right\|_{q}=1\right)$, and it suffices to show that $(2 \geqslant)\left\|\pi(a)+\pi\left(a_{n}\right)\right\|_{q}$ tends to 2 as $n$ goes to $\infty$ (due to Proposition 2.2).

We first notice that

$$
\begin{aligned}
\langle\pi(a), a\rangle & =\left\langle|a|^{p / q} u^{*}, u|a|\right\rangle=\operatorname{tr}\left(|a|^{p / a} u^{*} u|a|\right) \\
& =\operatorname{tr}\left(|a|^{p / q+1}\right)=\operatorname{tr}\left(|a|^{p}\right)=\|a\|_{p}^{p}=1,
\end{aligned}
$$

and $\left\langle\pi\left(a_{n}\right), a_{n}\right\rangle=1$, similarly. Thus, Hölder's inequality shows

$$
\left\|\pi(a)+\pi\left(a_{n}\right)\right\|_{q} \geqslant\left|\left\langle\pi(a)+\pi\left(a_{n}\right), a\right\rangle\right|=\left|1+\left\langle\pi\left(a_{n}\right), a\right\rangle\right| .
$$

Another use of Hölder's inequality shows that, for a small $\varepsilon>0$, we have

$$
\varepsilon \geqslant\left|\left\langle\pi\left(a_{n}\right), a-a_{n}\right\rangle\right|=\left|\left\langle\pi\left(a_{n}\right), a\right\rangle-1\right| \quad \text { (for } n \text { large enough). }
$$

The above two estimates mean that

$$
\left\|\pi(a)+\pi\left(a_{n}\right)\right\|_{q} \geqslant|2+z| \quad \text { with }|z| \leqslant \varepsilon
$$

(here, $z=\left\langle\pi\left(a_{n}\right), a\right\rangle-1 \in \mathbf{C}$ ). In particular, $\left\|\pi(a)+\pi\left(a_{n}\right)\right\|_{q} \geqslant 2-\varepsilon$ for $n$ large enough. Q.E.D.

The next result, which will be used later, might be of independent interest.

TheOREM 4.2. For $1 \leqslant p, p^{\prime}<\infty$, the map

$$
a \in L^{p^{\prime}}\left(\Re ; \phi_{0}\right)_{+} \rightarrow a^{p^{\prime} / p} \in L^{p}\left(\Re ; \phi_{0}\right)_{+}
$$

is a homeomorphism with respect to the norm topologies. 
Proof. Bijectivity is trivial so we must prove bicontinuity. Clearly we may assume $p^{\prime}=1$. Having proved Lemma 3.4, we need only prove continuity of the map $\left.a \in L^{p}\left(\cdot \pi ; \phi_{0}\right)_{+} \rightarrow a^{p} \in L^{1}(\cdot) ; \phi_{0}\right)_{+}$. However, this follows from Lemma 4.1 and Hölder's inequality due to $\pi(a) a=a^{p / q} a=a^{p}$. Q.E.D.

Lemma 4.3. For $2<p<\infty$, the maps

$$
\left.a \in L^{p}\left(\mathfrak{o} ; \phi_{0}\right) \rightarrow|a|=\left(a^{*} a\right)^{1 / 2} \in L^{p}(\Re) ; \phi_{0}\right)_{+}
$$

and

$$
\left.a \in L^{p}\left(\Re ; \phi_{0}\right) \rightarrow\left|a^{*}\right|=\left(a a^{*}\right)^{1 / 2} \in L^{p}(\Re) ; \phi_{0}\right)_{+}
$$

are norm-continuous.

Proof. When $\left\{a_{n}\right\}$ tends to $a$ in $L^{p}\left(\mathscr{N} ; \phi_{0}\right), a_{n}^{*}$ tends to $a^{*}$ so that $a_{n}^{*} a_{n}$ (resp. $\left.a_{n} a_{n}^{*}\right)$ tends to $a^{*} a$ (resp. $\left.a a^{*}\right)$ in $L^{p^{\prime}}(\mathfrak{N} ; \phi), 1<p^{\prime}=p / 2<\infty$, by Hölder's inequality. The lemma thus follows from Theorem 4.2. Q.E.D.

Here is the main result of the section.

Theorem 4.4. For $1 \leqslant p \leqslant \infty$, the "absolute value part" map $a \in L^{p}($ ( $\left.) ; \phi_{0}\right) \rightarrow$ $|a|=\left(a^{*} a\right)^{1 / 2} \in L^{p}\left(\mathfrak{N} ; \phi_{0}\right)_{+}$is norm-continuous.

Before proving it, we note that continuity is known for $p=1$, that is, for $)_{*}^{+}$(see [28] for example) and for $p=\infty$. Also, for $p=2$, the Powers-Størmer inequality [1, 9] states

$$
\||a|-|b|\|_{2}^{2} \leqslant\|a+b\|_{2}\|a-b\|_{2}, \quad a, b \in L^{2}\left(\mathfrak{O} \pi ; \phi_{0}\right) .
$$

Actually, the better estimate

$$
\||a|-|b|\|_{2} \leqslant \sqrt{2}\|a-b\|_{2}
$$

was obtained in [4]. We also remark that the result is proved for $C_{p}$-ideals in [24] (by a different method).

PROOF OF THE THEOREM. Thanks to the above remarks and Lemma 4.3, we assume $1<p<2$ in what follows. For $a=u|a| \in L^{p}\left(\mathfrak{N} ; \phi_{0}\right)$, we notice that

$$
\begin{gathered}
\pi(a)=|a|^{p / q} u^{*} \in L^{q}\left(\mathfrak{N} ; \phi_{0}\right), \quad 2<q<\infty, \\
\left(\pi(a) \pi(a)^{*}\right)^{1 / 2}=|a|^{p / q} \in L^{q}\left(\mathfrak{N} ; \phi_{0}\right)_{+}, \\
\left(|a|^{p / q}\right)^{q / p}=|a| \in L^{p}\left(\mathfrak{N} ; \phi_{0}\right)_{+} .
\end{gathered}
$$

Thus, the desired continuity follows from Lemma 4.1, (the second half of) Lemma 4.3, and Theorem 4.2. Q.E.D.

5. A one-parameter family of positive cones. As applications of Proposition 3.7 and Theorem 4.4, we prove the results on positive cones stated in $\$ 1$.

We recall that, for each $0 \leqslant \alpha \leqslant 1 / 2, P^{\alpha}\left(=P_{\phi_{0}}^{\alpha}\right)$ is the closure of the positive cone $\Delta^{\alpha} \Re_{+} \xi_{0}$ in the standard Hilbert space $\mathfrak{H}$ [1]. Improving previous results in [1, $3,13,16,25,27]$, we prove 
THEOREM 5.1. For each $0 \leqslant \alpha \leqslant 1 / 4$, the map $\xi \in P^{\alpha} \rightarrow \omega_{\xi} \in \mathfrak{M}_{*}^{+}$is a homeomorphism with respect to the norm topologies in $\mathcal{H}$ and $\mathfrak{M}_{*}$. If $\mathscr{N}$ is finite, then the result remains valid for each $0 \leqslant \alpha \leqslant 1 / 2$.

In both statements bijectivity is known [13], while continuity is trivial. We thus show the continuity of the inverse mapping.

To prove the first half of the theorem, we prepare the following lemma.

LEMMA 5.2. For each $a$ in $L^{p}\left(\mathfrak{T} ; \phi_{0}\right), 1 \leqslant p \leqslant \infty$ (which is identified with $\left.a \Delta^{1 / q} \in L^{1}(\Re) ; \phi_{0}\right)$; see 2$)$, we set

$$
f_{x}(a)=\left(a \Delta^{1 / q} \xi_{0} \mid x \xi_{0}\right)=\left(a \xi_{0} \mid x \xi_{0}\right), \quad x \in \mathscr{M} .
$$

Then we have $\left|f_{x}(a)\right| \leqslant\|a\|_{p}\|x\|$.

PROOF. We note that, for each $a=u \Delta_{\phi \phi_{0}}^{1 / p}, f_{x}(a)$ certainly makes sense since

$$
\begin{aligned}
f_{x}(a)= & \left(u \Delta_{\phi \phi_{0}}^{1 / p} \xi_{0} \mid x \xi_{0}\right)=\left(\Delta_{\phi \phi_{0}}^{1 / 2 p} \xi_{0} \mid \Delta_{\phi \phi_{0}}^{1 / 2 p} u^{*} x \xi_{0}\right), \\
& \mathbb{M} \xi_{0} \subseteq \mathcal{D}\left(\Delta_{\phi \phi_{0}}^{1 / 2 p}\right) \subseteq \mathscr{D}\left(\Delta_{\phi \phi_{0}}^{1 / 2 p}\right),
\end{aligned}
$$

and $f_{x}(a)$ is linear in $a$.

Thanks to Proposition 2.1, to complete the proof it suffices to check the inequality for the two extreme values of $p$, that is, $p=\infty$ and $p=1$. For $p=\infty$, we obviously have

$$
\left|\left(a \xi_{0} \mid x \xi_{0}\right)\right| \leqslant\|a\|\|x\| \quad\left(\|a\|=\|a\|_{\infty}\right)
$$

due to $\left\|\xi_{0}\right\|=1$, while for $p=1$ and $a=u \Delta_{\phi \phi_{0}}$ we estimate

$$
\begin{aligned}
\left|\left(u \Delta_{\phi \phi_{0}} \xi_{0} \mid x \xi_{0}\right)\right| & =\left|\left(\Delta_{\phi \phi_{0}}^{1 / 2} \xi_{0} \mid \Delta_{\phi \phi_{0}}^{1 / 2} u^{*} x \xi_{0}\right)\right|=\left|\left(\xi_{\phi} \mid J x^{*} u \xi_{\phi}\right)\right| \\
& =\left|\left(x^{*} u \xi_{\phi} \mid \xi_{\phi}\right)\right|=\left|\phi\left(x^{*} u\right)\right| \leqslant\|\phi\|\left\|x^{*}\right\|\|u\|=\|a\|_{1}\|x\| \text {. Q.E.D. }
\end{aligned}
$$

Proof of Theorem $5.1(0 \leqslant \alpha \leqslant 1 / 4)$. For a generic $\phi \in \Re_{*}^{+}$, a unique implementing vector $\zeta_{\phi}\left(=\zeta_{\phi, \alpha}\right)$ in $P^{\alpha}$ is explicitly given by

$$
\zeta_{\phi}=J\left|\Delta_{\phi \phi_{0}}^{1 / 2} \Delta^{1 / 2-2 \alpha}\right| \xi_{0} .
$$

(A homogeneity argument shows $\zeta_{\phi} \in P^{\alpha}$, while $\phi=\omega_{\zeta_{\phi}}$ follows from the fact that the phase part of $\Delta_{\phi \phi_{0}}^{1 / 2} \Delta^{1 / 2-2 \alpha}$ belongs to $\Re$. See [14] for details, especially the remark before Theorem 4.5 in [14].)

We now assume $\left\{\phi_{n}\right\}$ in $\mathscr{N}_{*}^{+}$tends to $\phi$ in norm. Being a Hilbert space, $\mathscr{H}$ is certainly a uniformly convex Banach space (the parallelogram law). We also have

$$
\lim _{n \rightarrow \infty}\left\|\zeta_{\phi_{n}}\right\|=\lim _{n \rightarrow \infty} \phi_{n}(1)^{1 / 2}=\phi(1)^{1 / 2}=\left\|\zeta_{\phi}\right\|
$$

By virtue of Lemma 3.2 it suffices to show that $\left(J \zeta_{\phi_{n}} \mid x \xi_{0}\right)=\left(\left|\Delta_{\phi_{n} \phi_{0}}^{1 / 2-2 \alpha}\right| \xi_{0} \mid x \xi_{0}\right)$ tends to $\left(J \zeta_{\phi} \mid x \xi_{0}\right)=\left(\left|\Delta_{\phi \phi_{0}}^{1 / 2} \Delta^{1 / 2-2 \alpha}\right| \xi_{0} \mid x \xi_{0}\right)$ for each fixed $x \in \mathscr{N}$. The PowersStørmer inequality and Hölder's inequality show that $\Delta_{\phi_{n} \phi_{0}}^{1 / 2} \Delta^{1 / 2-2 \alpha}$ tends to $\Delta_{\phi \phi_{0}}^{1 / 2} \Delta^{1 / 2-2 \alpha}$ in the $L^{p}$-norm (with $1 / p=1-2 \alpha$ ). Thus, the desired convergence follows from Lemma 5.2. Q.E.D. 
Proof of Theorem 5.1 ( $(9)$ is finite and $0 \leqslant \alpha \leqslant 1 / 2$ ). Let $\tau$ be a faithful tracial state on $\Re$, and $\left.L^{2}(\Re) \tau\right)$ the corresponding $L^{2}$-space (see $\left.\S 2\right)$. We recall that all

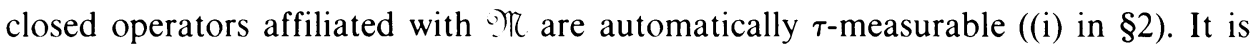
easy to observe that $\left.\left(\mathcal{M}, L^{2}(\Re) ; \tau\right),{ }^{*}, L^{2}(\because \eta ; \tau)_{+}\right)$is a standard form. Here, $\Re$ should be understood to act on $L^{2}(\mathcal{O}) \tau$ ) as left multiplications. By the uniqueness of a standard form $[\mathbf{1}, 9]$, we may and do identify

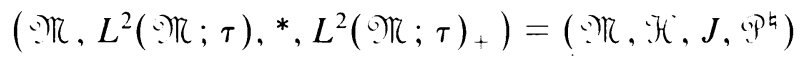

in the rest of the proof.

For a generic $\phi \in \mathrm{O}_{*}^{+}$, we denote its Radon-Nikdoym derivative $d \phi / d \tau$ relative to $\tau$ by $h_{\phi}$ (and $h_{\phi_{0}}=h_{0}$ for simplicity). In other words, a unique implementing vector $\xi_{\phi}$ in $\left.\rho^{\circ}=L^{2}(\Re) ; \tau\right)_{+}$is $h_{\phi}^{1 / 2}$. Then a unique implementing vector $\zeta_{\phi}$ $\left(=\zeta_{\phi, \alpha}\right)$ in $\left.P^{\alpha}\left(\subseteq L^{2}(\mathcal{O}) \tau ; \tau\right)\right)$ is explicitly given by

$$
\zeta_{\phi}=h_{0}^{2 \alpha-1 / 2}\left|h_{\phi}^{1 / 2} h_{0}^{1 / 2-2 \alpha}\right|=h_{0}^{2 \alpha-1 / 2}\left(h_{0}^{1 / 2-2 \alpha} h_{\phi} h_{0}^{1 / 2-2 \alpha}\right)^{1 / 2} .
$$

(In fact, since $\left.\zeta_{\phi} \zeta_{\phi}^{*}=h_{\phi}, \zeta_{\phi} \in L^{2}(\mathcal{O}) \tau ; \tau\right)$ and $\phi=\omega_{\zeta_{\phi}}$. Also, $h_{0}^{1 / 2-2 \alpha \zeta_{\phi}} \geqslant 0$ shows that $\zeta_{\phi} \in P^{\alpha}$. See $[13, \S 14]$ for details.)

We now assume $\left\{\phi_{n}\right\}$ in $\mathcal{M}_{*}^{+}$tends to $\phi$ in norm. Thus, $\left|\zeta_{\phi_{n}}^{*}\right|^{2}=h_{\phi_{n}}$ tends to $\left|\zeta_{\phi}^{*}\right|^{2}=h_{\phi}$ in the $L^{1}$-norm. To conclude that $\zeta_{\phi_{n}}$ tends to $\zeta_{\phi}$ (or equivalently, $\zeta_{\phi_{n}}^{*}$ tends to $\zeta_{\phi}^{*}$ ) in the $L^{2}$-norm, it suffices to show that $\zeta_{\phi_{n}}^{*}$ tends to $\zeta_{\phi}^{*}$ in measure (due to Proposition 3.7). The Powers-Størmer inequality yields that $h_{\phi_{n}}^{1 / 2}$ tends to $h_{\phi}^{1 / 2}$ in the $L^{2}$-norm, hence in measure ((vi) in $\left.\S 2\right)$. For a probability gage $\tau$, it is known [21] that the map $a \rightarrow|a|=\left(a^{*} a\right)^{1 / 2}$ is continuous with respect to the measure topology. Thus, $\zeta_{\phi_{n}}^{*}=\left|h_{\phi_{n}}^{1 / 2} h_{0}^{1 / 2-2 \alpha}\right| h_{0}^{2 \alpha-1 / 2}$ tends to $\zeta_{\phi}^{*}=\left|h_{\phi}^{1 / 2} h_{0}^{1 / 2-2 \alpha}\right| h_{0}^{2 \alpha-1 / 2}$ in measure due to $(v)$ in $\S 2$. Q.E.D.

6. $L^{p}$-norm inequalities. Our main concern here is to obtain necessary and sufficient conditions for certain $L^{p}$-norm inequalities to become equalities.

For $L^{p}$-spaces associated with a semifinite von Neumann algebra (and a trace on it), several $L^{p}$-norm inequalities are known. Although generic elements in these $L^{p}$-spaces are unbounded operators, one has to deal with just bounded operators due to the fact that they form a dense subspace ((i), (iii)' in \$2). On the other hand, in our $L^{p}$-spaces, all (nonzero) elements are unbounded so slightly different arguments are sometimes required. However, fortunately, we need not distinguish $a b=b a$ and the strong commutativity between $a$ and $b$ (Lemma 2.4).

Lemma 6.1. For $1<p<\infty$ and $0 \leqslant \lambda \leqslant 1$, we have:

(i) $1 \geqslant \lambda^{p}+(1-\lambda)^{p}$ and the equality holds if and only if $\lambda=0$ or 1 ;

(ii) $(1 / 2)^{p} \leqslant \frac{1}{2}\left\{\lambda^{p}+(1-\lambda)^{p}\right\}$ and the equality holds if and only if $\lambda=1 / 2$.

The proof is elementary so details are left to the reader.

Lemma 6.2. For commuting $a, b \in L^{p}\left(\mathfrak{N} ; \phi_{0}\right)_{+}, 1<p<\infty$, we have:

(i) $\operatorname{tr}\left((a+b)^{p}\right)=\operatorname{tr}\left(a^{p}+b^{p}\right)$ if and only if $a b=0$;

(ii) $\operatorname{tr}\left(\left\{\frac{1}{2}(a+b)\right\}^{p}\right)=\frac{1}{2} \operatorname{tr}\left(a^{p}+b^{p}\right)$ if and only if $a=b$. 
Proof. To prove it we assume $a=\Delta_{\phi \phi_{0}}^{1 / p}$ and $b=\Delta_{\psi \phi_{0}}^{1 / p}\left(\phi \cong a^{p}, \psi \cong b^{p} \in \mathbb{R}_{*}^{+}\right)$. Furthermore, since $L^{p}\left(\mathfrak{N} ; \phi_{0}\right)$ does not depend on a choice of $\phi_{0}$ up to a Banach space isometry $[\mathbf{1 0}, \mathbf{1 5}]$ (after cutting the algebra), we may and do assume

$$
\Delta_{\phi \phi_{0}}^{1 / p}+\Delta_{\psi \phi_{0}}^{1 / p}=\Delta^{1 / p} \quad\left(=\Delta_{\phi_{0} \phi_{0}}^{1 / p}\right)
$$

Notice that all the operators here mutually commute and $\Delta_{\phi \phi_{0}}^{1 / p} \leqslant \Delta^{1 / p}, \Delta_{\psi \phi_{0}}^{1 / p} \leqslant \Delta^{1 / p}$. Thus, one can pick up positive operators $h, k$ in $\Re$ such that $\Delta_{\phi \phi_{0}}^{1 / 2 p}=\sqrt{h} \Delta^{1 / 2 p}$, $\Delta_{\psi \phi_{0}}^{1 / 2 p}=\sqrt{k} \Delta^{1 / 2 p}$ and $h, k$ commute with $\Delta$. Since

$$
\Delta^{1 / p}=\Delta_{\phi \phi_{0}}^{1 / p}+\Delta_{\psi \phi_{0}}^{1 / p}=(h+k) \Delta^{1 / p}
$$

and $\Delta$ has a dense range, we get $h+k=1$ and

$$
\Delta_{\phi \phi_{0}}^{z}=h^{p z} \Delta^{z}, \quad \Delta_{\psi \phi_{0}}^{z}=(1-h)^{p z} \Delta^{z}, \quad \operatorname{Re} z \geqslant 0 .
$$

Using the spectral decomposition $h=\int_{0}^{1} \lambda d e_{\lambda}$ and $d \mu(\lambda)=d\left\|e_{\lambda} \xi_{0}\right\|^{2}$, we compute

$$
\begin{aligned}
\operatorname{tr}\left(a^{p}+b^{p}\right) & =\left(\left\{h^{p}+(1-h)^{p}\right\} \Delta \xi_{0} \mid \xi_{0}\right)=\left(\left\{h^{p}+(1-h)^{p}\right\} \xi_{0} \mid \xi_{0}\right) \\
& =\int_{0}^{1}\left\{\lambda^{p}+(1-\lambda)^{p}\right\} d \mu(\lambda), \\
\operatorname{tr}\left((a+b)^{p}\right) & =\left(\Delta \xi_{0} \mid \xi_{0}\right)=\left(\xi_{0} \mid \xi_{0}\right)=\int_{0}^{1} d \mu(\lambda) .
\end{aligned}
$$

It follows from Lemma 6.1(i) that the two integrals are equal if and only if $d \mu(\lambda)$ is supported on the finite sets $\{0,1\}$

$$
\begin{aligned}
& \Leftrightarrow \phi_{0}\left(h-h^{2}\right)=\int_{0}^{1}\left(\lambda-\lambda^{2}\right) d \mu(\lambda)=0 \\
& \Leftrightarrow h(1-h)=0 \\
& \Leftrightarrow h \Delta^{1 / p}(1-h) \Delta^{1 / p}=a b=0 .
\end{aligned}
$$

The second assertion is obtained by similar arguments together with Lemma 6.1(ii). Q.E.D.

Proposition 6.3. For $a, b \in L^{p}\left(\Re ; \phi_{0}\right)_{+}, 1<p<\infty$, we have

$$
\operatorname{tr}\left(a^{p}+b^{p}\right) \leqslant \operatorname{tr}\left((a+b)^{p}\right) .
$$

Equality holds if and only if $a b=0$.

Proof. The first part is exactly Lemma 3.3. Also, if $a b=0$, we have $\operatorname{tr}\left(a^{p}+b^{p}\right)$ $=\operatorname{tr}\left((a+b)^{p}\right)$. Conversely, we now assume this, thanks to Lemma 6.2(i), it suffices to show $[a, b]=0$. Fix an arbitrary selfadjoint $x$ in $\mathscr{N}$ and set

$$
f(t)=\operatorname{tr}\left(\left(a+e^{i t x} b e^{-i t x}\right)^{p}\right), \quad t \in \mathbf{R} .
$$

The first half of the proposition and the fact that $e^{i t x}$ is a unitary imply

$$
f(t) \geqslant \operatorname{tr}\left(a^{p}+e^{i t x} b^{p} e^{-i t x}\right)=\operatorname{tr}\left(a^{p}+b^{p}\right)=f(0) .
$$

On the other hand, the map $t \in \mathbf{R} \rightarrow e^{i t x} b e^{-i t x} \in L^{p}\left(\mathscr{N} ; \phi_{0}\right)$ is differentiable and its derivative at 0 is $i[x, b]$. Thus it follows from Lemma 3.1 that

$$
0=i p \operatorname{tr}\left((a+b)^{p-1}[x, b]\right)=i p \operatorname{tr}\left(\left\{(a+b)^{p-1} b-b(a+b)^{p-1}\right\} x\right) .
$$


(Although Lemma 3.1 is not applicable when $[x, b]=0,[x, b]=0$ implies $f(t)$ is constant and we get the above equality anyway.) Thanks to the duality o $\pi=\Omega_{*}^{*}=$ $L^{1}\left(\mathfrak{O} ; \phi_{0}\right)^{*}$ and $\left.\mathfrak{N}=\mathcal{O} \mathbb{R}_{s a}+i\right)_{s a}$, we have $\left[(a+b)^{p-1}, b\right]=0$ so $b$ commutes with $a+b$ and $a$. Q.E.D.

Proposition 6.4. For $a, b \in L^{p}(\mathcal{O})$; $\left.\phi_{0}\right)_{+}, 1<p<\infty$, we have

$$
\operatorname{tr}\left((a+b)^{p}\right) \leqslant 2^{p-1}\left(a^{p}+b^{p}\right),
$$

or, equivalently,

$$
\operatorname{tr}\left(\left\{\frac{1}{2}(a+b)\right\}^{p}\right) \leqslant \frac{1}{2} \operatorname{tr}\left(a^{p}+b^{p}\right) .
$$

Equality holds if and only if $a=b$.

Proof. The triangle inequality for \|\|$_{p}$ and the convexity of $t^{p}, t \geqslant 0$, imply

$$
\begin{aligned}
\operatorname{tr}\left((a+b)^{p}\right) & =\|a+b\|_{p}^{p} \leqslant\left(\|a\|_{p}+\|b\|_{p}\right)^{p} \\
& \leqslant 2^{p-1}\left(\|a\|_{p}^{p}+\|b\|_{p}^{p}\right)=2^{p-1} \operatorname{tr}\left(a^{p}+b^{p}\right) .
\end{aligned}
$$

Also,

$$
\operatorname{tr}\left((a+b)^{p}\right)=2^{p^{-1}} \operatorname{tr}\left(a^{p}+b^{p}\right) \quad \text { if } a=b .
$$

Conversely, if this equality holds, arguments similar to those in the proof of 6.3 (based on $\left.f(t)=\operatorname{tr}\left(\left\{\frac{1}{2}\left(a+e^{i t x} b e^{-i t x}\right)^{p}\right\}\right) \leqslant f(0)\right)$ show $[a, b]=0$. Thus, $a=b$ follows from Lemma 6.2(ii). Q.E.D.

Combining the preceding two propositions, we get

Corollary 6.5. For $a, b \in L^{p}\left(\mathfrak{N} ; \phi_{0}\right)_{+}, 1<p<\infty$, we have

$$
2^{1-p}\|a+b\|_{p}^{p} \leqslant\|a\|_{p}^{p}+\|b\|_{p}^{p} \leqslant\|a+b\|_{p}^{p} .
$$

Here, the first (resp. second) inequality becomes an equality if and only if $a=b$ (resp. $a b=0$ ).

Recall that we did not use Clarkson's (nor McCarthy's) inequality to show the uniform convexity of noncommutative $L^{P}$-spaces (Proposition 2.2). We now prove Clarkson's inequality and examine when it reduces to an equality.

For $2<p<\infty$ with $\left.1<p^{\prime}=p / 2<\infty, a, b \in L^{p}(\mathcal{O}) ; \phi_{0}\right)$, we set

$$
c=a^{*} a+b^{*} b \in L^{p^{\prime}}\left(\text { OM } ; \phi_{0}\right), \quad b=a^{*} b+b^{*} a \in L^{p^{\prime}}\left(\text { 유 } ; \phi_{0}\right) \text {. }
$$

We estimate

$$
\begin{aligned}
2\left(\|a\|_{p}^{p}+\|b\|_{p}^{p}\right) & =2 \operatorname{tr}\left(\left(a^{*} a\right)^{p^{\prime}}+\left(b^{*} b\right)^{p^{\prime}}\right) \\
& \leqslant 2 \operatorname{tr}\left(\left(a^{*} a+b^{*} b\right)^{p^{\prime}}\right) \quad \text { (the first half of Proposition 6.3) } \\
& =2\|c\|_{p^{\prime}}^{p^{\prime}} \leqslant 2\left\{\frac{1}{2}\|c+d\|_{p^{\prime}}+\frac{1}{2}\|c-d\|_{p^{\prime}}\right\}^{p^{\prime}} \\
& \left.\quad \text { (the triangle inequality for }\|\|_{p^{\prime}}\right) \\
& \leqslant\|c+d\|_{p^{\prime}}^{p^{\prime}}+\|c-d\|_{p^{\prime}}^{p^{\prime}} \quad\left(\text { convexity of } t^{p^{\prime}}, t \geqslant 0\right) \\
& =\operatorname{tr}\left(|c+d|^{p^{\prime}}\right)+\operatorname{tr}\left(|c-d|^{p^{\prime}}\right)=\operatorname{tr}\left(|a+b|^{p}\right)+\operatorname{tr}\left(|a-b|^{p}\right) \\
& =\|a+b\|_{p}^{p}+\|a-b\|_{p}^{p} .
\end{aligned}
$$


The next result was obtained in [30] for $L^{p}$-spaces arising from a semifinite von Neumann algebra and bounded $a, b$.

Theorem 6.6 (Clarkson's inequality). For $a, b$ in $L^{p}\left(\mathfrak{O} ; \phi_{0}\right), 2<p<\infty$, we have

$$
2\left(\|a\|_{p}^{p}+\|b\|_{p}^{p}\right) \leqslant\|a+b\|_{p}^{p}+\|a-b\|_{p}^{p}
$$

or, equivalently,

$$
\|a+b\|_{p}^{p}+\|a-b\|_{p}^{p} \leqslant 2^{p-1}\left(\|a\|_{p}^{p}+\|b\|_{p}^{p}\right) .
$$

Furthermore, equality holds in the first inequality if and only if $a b^{*}=b^{*} a=0$.

Proof. We have already shown the first half. We now assume equality holds. In particular, the very first inequality in the estimates right before the theorem reduces to an equality. Hence, the second half of Proposition 6.3 yields

$$
a^{*} a b^{*} b=|a|^{2}|b|^{2}=0 \text {. }
$$

Thus, $|a|$ and $|b|$ commute and $|a||b|=\left(|a|^{2}|b|^{2}\right)^{1 / 2}=0$. Considering the polar decomposition of $a$ and $b$, we get $a b^{*}=0$. The adjoint operation being isometric in each $L^{p}$-space, one still gets equality in Clarkson's inequality after $(a, b)$ is replaced by $\left(a^{*}, b^{*}\right)$. We thus get $b^{*} a=\left(\left(a^{*}\right)\left(b^{*}\right)^{*}\right)^{*}=0^{*}=0$.

Conversely, if $a b^{*}=b^{*} a=0$, then we get

$$
|a \pm b|^{2}=(a \pm b)^{*}(a \pm b)=a^{*} a+b^{*} b=|a|^{2}+|b|^{2} \text {. }
$$

Also, $a b^{*}=0$ implies $|a||b|=0$. We thus have

$$
|a \pm b|^{p}=\left(|a|^{2}+|b|^{2}\right)^{p / 2}=|a|^{p}+|b|^{p}, \quad\|a \pm b\|_{p}^{p}=\|a\|_{p}^{p}+\|b\|_{p}^{p}
$$

Thus, we get equality in Clarkson's inequality. Q.E.D.

\section{REFERENCES}

1. H. Araki, Some properties of modular conjugation operator of a von Neumann algebra and a non-commutative Radon-Nikodym theorem with a chain rule, Pacific J. Math. 50 (1974), 309-354.

2. __ Relative entropy for states of von Neumann algebras. II, Publ. Res. Inst. Math. Sci. 13 (1977), 173-194.

3. H. Araki and T. Masuda, Positive cones and $L^{p}$-spaces for von Neumann algebras, Publ. Res. Inst. Math. Sci. 18 (1982), 339-411.

4. H. Araki and S. Yamagami, An inequality for Hilbert-Schmidt norm, Comm. Math. Phys. 81 (1981), $89-96$

5. J. Bergh and J. Löfström, Interpolation spaces, an introduction, Springer-Verlag, Berlin and New York, 1976.

6. A. P. Calderón, Intermediate spaces and the interpolation: the complex method, Studia Math. 24 (1964), 113-190.

7. A. Connes, Une classification des facteurs de type III, Ann. Sci. École Norm. Sup. (4) 6 (1973), $133-252$.

8. __ On the spatial theory of von Neumann algebras, J. Funct. Anal. 35 (1980), 153-164.

9. U. Haagerup, The standard form of von Neumann algebras, Math. Scand. 37 (1975), 271-283.

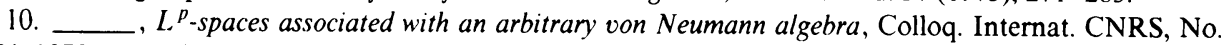
274, 1979, pp. 175-184.

11. F. Hansen, An operator inequality, Math. Ann. 246 (1980), 249-250.

12. , Les espaces $L^{p} d$ 'une algèbre de von Neumann, J. Funct. Anal. 40 (1981), 151-169. 
13. H. Kosaki, Positive cones associated with a von Neumann algebra, Math. Scand. 47 (1980), 295-307.

14. , Positive cones and $L^{P}$-spaces associated with a von Neumann algebra, J. Operator Theory 6 (1981), 13-23.

15. tive $L^{p}$-spaces), J. Funct. Anal. (to appear).

16. __ Remarks on positive cones associated with a von Neumann algebra. Tôhoku Math. J. (2) 33 (1981), 587-591.

17. G. Köthe, Topological vector spaces. I, Springer-Verlag, Berlin and New York, 1969.

18. R. Kunze, $L^{p}$-Fourier transforms on locally compact unimodular groups, Trans. Amer. Math. Soc. 89 (1958), 519-540.

19. C. A. McCarthy, $C p$, Israel J. Math. 5 (1967), 249-271.

20. E. Nelson, Notes on non-commutative integration, J. Funct. Anal. 15 (1974), 104-116.

21. A. R. Padmanabhan, Probabilistic aspects of a von Neumann algebra, J. Funct. Anal. 31 (1979), $139-149$.

22. M. Reed and B. Simon, Methods of modern mathematical physics, Vols. I, II, Academic Press, New York, 1972, 1975.

23. I. Segal, A non-commutative extension of abstract integration, Ann. of Math. (2) 37 (1953), 401-457.

24. B. Simon, Trace ideals and their applications, Cambridge Univ. Press, London, 1979.

25. C. Skau, Positive self-adjoint extensions of operators affiliated with a von Neumann algebra, Math. Scand. 44 (1979), 171-195.

26. F. Stinespring, Integration theorems for gages and duality theorems for unimodular groups, Trans. Amer. Math. Soc. 90 (1959), 15-56.

27. M. Takesaki, Tomita's theory of modular Hilbert algebras and its applications, Lecture Notes in Math., vol. 128, Springer-Verlag, Berlin and New York, 1970.

28. Theory of operator algebras. I. Springer-Verlag, Berlin and New York, 1979.

29. P. K. Tam, Isometries of $L^{p}$-spaces associated with semi-finite von Neumann algebras, Trans. Amer. Math. Soc. 254 (1979), 339-354.

30. M. Terp, $L^{p}$-spaces associated with von Neumann algebras, preprint.

31. F. J. Yeadon, Convergence of measurable operators, Proc. Cambridge Philos. Soc. 74 (1973), $257-268$.

Department of Mathematics, Purdue University, West Lafayette, Indiana 47907

Current address: Department of Mathematics, University of Pennsylvania, Philadelphia, Pennsylvania 19104-3859 